\title{
Remarks on G-metric spaces and fixed point theorems
}

\author{
Mohamed Jleli and Bessem Samet ${ }^{*}$
}

"Correspondence: bsamet@ksu.edu.sa Department of Mathematics, King Saud University, Riyadh, Saudi Arabia

\begin{abstract}
We discuss the introduced concept of $G$-metric spaces and the fixed point existing results of contractive mappings defined on such spaces. In particular, we show that the most obtained fixed point theorems on such spaces can be deduced immediately from fixed point theorems on metric or quasi-metric spaces.
\end{abstract}

MSC: 47H10; $11 \mathrm{~J} 83$

Keywords: G-metric; quasi-metric; metric; fixed point

\section{Introduction}

In 2005, Mustafa and Sims introduced a new class of generalized metric spaces (see [1, 2]), which are called $G$-metric spaces, as generalization of a metric space $(X, d)$. Subsequently, many fixed point results on such spaces appeared (see, for example, [3-7]).

Here, we present the necessary definitions and results in $G$-metric spaces, which will be useful for the rest of the paper. However, for more details, we refer to $[1,2]$.

Definition 1.1 Let $X$ be a nonempty set. Suppose that $G: X \times X \times X \rightarrow[0,+\infty)$ is a function satisfying the following conditions:

(1) $G(x, y, z)=0$ if and only if $x=y=z$;

(2) $0<G(x, x, y)$ for all $x, y \in X$ with $x \neq y$;

(3) $G(x, x, y) \leq G(x, y, z)$ for all $x, y, z \in X$ with $y \neq z$;

(4) $G(x, y, z)=G(x, z, y)=G(y, z, x)=\cdots$ (symmetry in all three variables);

(5) $G(x, y, z) \leq G(x, a, a)+G(a, y, z)$ for all $x, y, z, a \in X$.

Then $G$ is called a $G$-metric on $X$ and $(X, G)$ is called a $G$-metric space.

Definition 1.2 A $G$-metric space $(X, G)$ is said to be symmetric if $G(x, y, y)=G(y, x, x)$ for all $x, y \in X$.

Definition 1.3 Let $(X, G)$ be a $G$-metric space. We say that $\left\{x_{n}\right\}$ is

(1) a G-Cauchy sequence if, for any $\varepsilon>0$, there is $N \in \mathbb{N}$ (the set of all positive integers) such that for all $n, m, l \geq N, G\left(x_{n}, x_{m}, x_{l}\right)<\varepsilon$;

(2) a $G$-convergent sequence to $x \in X$ if, for any $\varepsilon>0$, there is $N \in \mathbb{N}$ such that for all $n, m \geq N, G\left(x, x_{n}, x_{m}\right)<\varepsilon$

A $G$-metric space $(X, G)$ is said to be complete if every $G$-Cauchy sequence in $X$ is $G$ convergent in $X$.

(c) 2012 Jleli and Samet; licensee Springer. This is an Open Access article distributed under the terms of the Creative Commons Attribution License (http://creativecommons.org/licenses/by/2.0), which permits unrestricted use, distribution, and reproduction in any medium, provided the original work is properly cited. 
Proposition 1.1 Let $(X, G)$ be a G-metric space. The following are equivalent:

(1) $\left\{x_{n}\right\}$ is G-convergent to $x$;

(2) $G\left(x_{n}, x_{n}, x\right) \rightarrow 0$ as $n \rightarrow+\infty$;

(3) $G\left(x_{n}, x, x\right) \rightarrow 0$ as $n \rightarrow+\infty$;

(4) $G\left(x_{n}, x_{m}, x\right) \rightarrow 0$ as $n, m \rightarrow+\infty$.

Proposition 1.2 Let $(X, G)$ be a G-metric space. Then the following are equivalent:

(1) the sequence $\left\{x_{n}\right\}$ is G-Cauchy;

(2) $G\left(x_{n}, x_{m}, x_{m}\right) \rightarrow 0$ as $n, m \rightarrow+\infty$.

An interesting observation is that any $G$-metric space $(X, G)$ induces a metric $d_{G}$ on $X$ given by

$$
d_{G}(x, y)=G(x, y, y)+G(y, x, x), \quad \text { for all } x, y \in X .
$$

Moreover, $(X, G)$ is $G$-complete if and only if $\left(X, d_{G}\right)$ is complete.

It was observed that in the symmetric case $((X, G)$ is symmetric), many fixed point theorems on G-metric spaces are particular cases of existing fixed point theorems in metric spaces. In this paper, we discuss the non-symmetric case. We will show that such spaces have a quasi-metric type structure and then many results on such spaces can be derived from fixed point theorems on quasi-metric spaces.

\section{Basic definitions and results}

As we mentioned earlier, G-metric spaces have a quasi-metric type structure. Indeed, we have the following result.

Theorem 2.1 Let $(X, G)$ be a G-metric space. The function $d: X \times X \rightarrow[0, \infty)$ defined by $d(x, y)=G(x, y, y)$ satisfies the following properties:

(1) $d(x, y)=0$ if and only if $x=y$;

(2) $d(x, y) \leq d(x, z)+d(z, y)$ for any points $x, y, z \in X$.

Proof The proof of (1) follows immediately from the property (1) in Definition 1.1. Now, let $x, y, z$ be any points in $X$. Using the property (5) in Definition 1.1, we have

$$
d(x, y)=G(x, y, y) \leq G(x, z, z)+G(z, y, y)=d(x, z)+d(z, y) .
$$

Thus, (2) holds.

The above result suggests the following definition.

Definition 2.1 Let $X$ be a nonempty set and $d: X \times X \rightarrow[0, \infty)$ be a given function which satisfies

(1) $d(x, y)=0$ if and only if $x=y$;

(2) $d(x, y) \leq d(x, z)+d(z, y)$ for any points $x, y, z \in X$.

Then $d$ is called a quasi-metric and the pair $(X, d)$ is called a quasi-metric space.

Note that any metric space is a quasi-metric space, but the converse is not true in general. Now, we define convergence and completeness on quasi-metric spaces. 
Definition 2.2 Let $(X, d)$ be a quasi-metric space, $\left\{x_{n}\right\}$ be a sequence in $X$, and $x \in X$. The sequence $\left\{x_{n}\right\}$ converges to $x$ if and only if

$$
\lim _{n \rightarrow \infty} d\left(x_{n}, x\right)=\lim _{n \rightarrow \infty} d\left(x, x_{n}\right)=0 .
$$

Definition 2.3 Let $(X, d)$ be a quasi-metric space and $\left\{x_{n}\right\}$ be a sequence in $X$. We say that $\left\{x_{n}\right\}$ is left-Cauchy if and only if for every $\varepsilon>0$ there exists a positive integer $N=N(\varepsilon)$ such that $d\left(x_{n}, x_{m}\right)<\varepsilon$ for all $n \geq m>N$.

Definition 2.4 Let $(X, d)$ be a quasi-metric space and $\left\{x_{n}\right\}$ be a sequence in $X$. We say that $\left\{x_{n}\right\}$ is right-Cauchy if and only if for every $\varepsilon>0$ there exists a positive integer $N=N(\varepsilon)$ such that $d\left(x_{n}, x_{m}\right)<\varepsilon$ for all $m \geq n>N$.

Definition 2.5 Let $(X, d)$ be a quasi-metric space and $\left\{x_{n}\right\}$ be a sequence in $X$. We say that $\left\{x_{n}\right\}$ is Cauchy if and only if for every $\varepsilon>0$ there exists a positive integer $N=N(\varepsilon)$ such that $d\left(x_{n}, x_{m}\right)<\varepsilon$ for all $m, n>N$.

Obviously, a sequence $\left\{x_{n}\right\}$ in a quasi-metric space is Cauchy if and only if it is leftCauchy and right-Cauchy.

Definition 2.6 Let $(X, d)$ be a quasi-metric space. We say that

(1) $(X, d)$ is left-complete if and only if each left-Cauchy sequence in $X$ is convergent;

(2) $(X, d)$ is right-complete if and only if each right-Cauchy sequence in $X$ is convergent;

(3) $(X, d)$ is complete if and only if each Cauchy sequence in $X$ is convergent.

The following result is an immediate consequence of the above definitions and results.

Theorem 2.2 Let $(X, G)$ be a G-metric space. Let $d: X \times X \rightarrow[0, \infty)$ be the function defined by $d(x, y)=G(x, y, y)$. Then

(1) $(X, d)$ is a quasi-metric space;

(2) $\left\{x_{n}\right\} \subset X$ is G-convergent to $x \in X$ if and only if $\left\{x_{n}\right\}$ is convergent to $x$ in $(X, d)$;

(3) $\left\{x_{n}\right\} \subset X$ is G-Cauchy if and only if $\left\{x_{n}\right\}$ is Cauchy in $(X, d)$;

(4) $(X, G)$ is $G$-complete if and only if $(X, d)$ is complete.

Every quasi-metric induces a metric, that is, if $(X, d)$ is a quasi-metric space, then the function $\delta: X \times X \rightarrow[0, \infty)$ defined by

$$
\delta(x, y)=\max \{d(x, y), d(y, x)\}
$$

is a metric on $X$.

The following result is an immediate consequence of the above definitions and results.

Theorem 2.3 Let $(X, G)$ be a G-metric space. Let $\delta: X \times X \rightarrow[0, \infty)$ be the function defined by $\delta(x, y)=\max \{G(x, y, y), G(y, x, x)\}$. Then

(1) $(X, \delta)$ is a metric space;

(2) $\left\{x_{n}\right\} \subset X$ is G-convergent to $x \in X$ if and only if $\left\{x_{n}\right\}$ is convergent to $x$ in $(X, \delta)$;

(3) $\left\{x_{n}\right\} \subset X$ is G-Cauchy if and only if $\left\{x_{n}\right\}$ is Cauchy in $(X, \delta)$;

(4) $(X, G)$ is G-complete if and only if $(X, \delta)$ is complete. 


\section{Discussion on fixed point results on G-metric spaces}

\subsection{From metric to G-metric: the linear case}

In this section, we show that in the case of linear contractive conditions, the existing fixed point results on $G$-metric spaces are immediate consequences of existing fixed point theorems on metric spaces.

As a model example, we consider the following result of Mustafa et al. [8].

Theorem 3.1 (Mustafa et al. [8]) Let $(X, G)$ be a complete G-metric space, and let $T: X \rightarrow$ $X$ be a mapping that satisfies the following condition: for all $x, y, z \in X$,

$$
G(T x, T y, T z) \leq a G(x, T x, T x)+b G(y, T y, T y)+c G(z, T z, T z)+d G(x, y, z)
$$

where $a, b, c, d$ are positive constants such that $k=a+b+c+d<1$. Then $T$ has a unique fixed point.

Now, we will show that the above result is an immediate consequence of Ćirić's fixed point theorem [9]. Indeed, taking $z=y$ in (3.1), we have

$$
G(T x, T y, T y) \leq k \max \{G(x, T x, T x), G(y, T y, T y), G(x, y, y)\},
$$

for all $x, y \in X$. Also, from (3.1), we have

$$
G(T y, T x, T x) \leq k \max \{G(y, T y, T y), G(x, T x, T x), G(y, x, x)\},
$$

for all $x, y \in X$. Define the metric space $\delta: X \times X \rightarrow[0, \infty)$ by

$$
\delta(x, y)=\max \{G(x, y, y), G(y, x, x)\} .
$$

It follows from (3.2) and (3.3) that

$$
\delta(T x, T y) \leq k \max \{\delta(x, T x), \delta(y, T y), \delta(x, y)\} .
$$

Now, $T$ satisfies Ćirićs contractive condition [9] in the complete metric space $(X, \delta)$ (see Theorem 2.3), then $T$ has a unique fixed point.

For more details about the linear case, we refer the reader to [10].

\subsection{From quasi-metric to $G$-metric: the nonlinear case}

In some cases, when the contractive condition is of nonlinear type, the above strategy cannot be used. However, we will show that we can deduce fixed point results on $G$-metric spaces from fixed point results on quasi-metric spaces. As a model example, we consider a weakly contractive condition. At first, we need the following fixed point theorem on quasi-metric spaces.

Theorem 3.2 Let $(X, d)$ be a complete quasi-metric space and $T: X \rightarrow X$ be a mapping satisfying

$$
d(T x, T y) \leq d(x, y)-\varphi(d(x, y))
$$


for all $x, y \in X$, where $\varphi:[0, \infty) \rightarrow[0, \infty)$ is continuous with $\varphi^{-1}(\{0\})=\{0\}$. Then $T$ has a unique fixed point.

Proof Let $x_{0}$ be any point in $X$ and $\left\{x_{n}\right\}$ be the sequence defined by $x_{n+1}=T x_{n}$ for all $n \geq 0$. From (3.4), we have

$$
d\left(x_{n}, x_{n+1}\right) \leq d\left(x_{n-1}, x_{n}\right)-\varphi\left(d\left(x_{n-1}, x_{n}\right)\right), \quad \text { for all } n \geq 1 .
$$

This implies that $\left\{d\left(x_{n}, x_{n+1}\right)\right\}$ is a decreasing sequence of positive numbers. Then there exists $r \geq 0$ such that $d\left(x_{n}, x_{n+1}\right) \rightarrow r$ as $n \rightarrow \infty$. Letting $n \rightarrow \infty$ in (3.5), we get that $\varphi(r)=$ 0 , that is, $r=0$. Thus, we have

$$
\lim _{n \rightarrow \infty} d\left(x_{n}, x_{n+1}\right)=0
$$

Using the same technique, we also have

$$
\lim _{n \rightarrow \infty} d\left(x_{n+1}, x_{n}\right)=0
$$

Now, we shall prove that $\left\{x_{n}\right\}$ is a Cauchy sequence in the quasi-metric space $(X, d)$, that is, $\left\{x_{n}\right\}$ is left-Cauchy and right-Cauchy. Suppose that $\left\{x_{n}\right\}$ is not a left-Cauchy sequence. Then there exists $\varepsilon>0$ for which we can find subsequences $\left\{x_{n(k)}\right\}$ and $\left\{x_{m(k)}\right\}$ of $\left\{x_{n}\right\}$ with $n(k)>m(k)>k$ such that

$$
d\left(x_{n(k)}, x_{m(k)}\right) \geq \varepsilon,
$$

for all $k$. Further, corresponding to $m(k)$, we can choose $n(k)$ such that it is the smallest integer with $n(k)>m(k)$ satisfying the above inequality. Then

$$
d\left(x_{n(k)-1}, x_{m(k)}\right)<\varepsilon,
$$

for all $k$. On the other hand, we have

$$
\varepsilon \leq d\left(x_{n(k)}, x_{m(k)}\right) \leq d\left(x_{n(k)}, x_{n(k)-1}\right)+d\left(x_{n(k)-1}, x_{m(k)}\right)<d\left(x_{n(k)}, x_{n(k)-1}\right)+\varepsilon .
$$

Letting $k \rightarrow \infty$ and using (3.7), we get

$$
\lim _{k \rightarrow \infty} d\left(x_{n(k)}, x_{m(k)}\right)=\varepsilon
$$

We have

$$
d\left(x_{n(k)-1}, x_{m(k)-1}\right) \leq d\left(x_{n(k)-1}, x_{n(k)}\right)+d\left(x_{n(k)}, x_{m(k)}\right)+d\left(x_{m(k)}, x_{m(k)-1}\right)
$$

and

$$
d\left(x_{n(k)}, x_{m(k)}\right) \leq d\left(x_{n(k)}, x_{n(k)-1}\right)+d\left(x_{n(k)-1}, x_{m(k)-1}\right)+d\left(x_{m(k)-1}, x_{m(k)}\right) .
$$


Letting $k \rightarrow \infty$ in the above inequalities, using (3.6), (3.7), and (3.8), we get

$$
\lim _{k \rightarrow \infty} d\left(x_{n(k)-1}, x_{m(k)-1}\right)=\varepsilon
$$

Now, from (3.4), for all $k$, we have

$$
d\left(x_{n(k)}, x_{m(k)}\right) \leq d\left(x_{n(k)-1}, x_{m(k)-1}\right)-\varphi\left(d\left(x_{n(k)-1}, x_{m(k)-1}\right)\right) .
$$

Letting $k \rightarrow \infty$ in the above inequality, using (3.8) and (3.9), we obtain

$$
\varepsilon \leq \varepsilon-\varphi(\varepsilon)
$$

which implies that $\varepsilon=0$ : a contradiction with $\varepsilon>0$. Then we proved that $\left\{x_{n}\right\}$ is a leftCauchy sequence. Similarly, we can show that $\left\{x_{n}\right\}$ is a right-Cauchy sequence. Then $\left\{x_{n}\right\}$ is a Cauchy sequence in the complete quasi-metric space $(X, d)$. This implies that there exists $a \in X$ such that

$$
\lim _{n \rightarrow \infty} d\left(x_{n}, a\right)=\lim _{n \rightarrow \infty} d\left(a, x_{n}\right)=0 .
$$

Now, we have

$$
d\left(x_{n}, T a\right)=d\left(T x_{n-1}, T a\right) \leq d\left(x_{n-1}, a\right)-\varphi\left(d\left(x_{n-1}, a\right)\right) .
$$

Letting $n \rightarrow \infty$ and using (3.10), we have

$$
\lim _{n \rightarrow \infty} d\left(x_{n}, T a\right)=0
$$

Similarly, we have

$$
d\left(T a, x_{n}\right)=d\left(T a, T x_{n-1}\right) \leq d\left(a, x_{n-1}\right)-\varphi\left(d\left(a, x_{n-1}\right)\right) .
$$

Letting $n \rightarrow \infty$ and using (3.10), we have

$$
\lim _{n \rightarrow \infty} d\left(T a, x_{n}\right)=0
$$

Then, we have

$$
\lim _{n \rightarrow \infty} d\left(x_{n}, T a\right)=\lim _{n \rightarrow \infty} d\left(T a, x_{n}\right)=0 .
$$

It follows from (3.10) and (3.11) that $a=T a$, that is, $a$ is a fixed point of $T$.

To show the uniqueness of the fixed point, suppose that $b$ is also a fixed point of $T$. From (3.4), we have

$$
d(a, b)=d(T a, T b) \leq d(a, b)-\varphi(d(a, b))
$$

which implies that $d(a, b)=0$, that is, $a=b$. Then $a$ is the unique fixed point of $T$. 
Now, from Theorem 3.2, we deduce immediately the following fixed point theorem on $G$-metric spaces.

Theorem 3.3 Let $(X, G)$ be a G-complete metric space and $T: X \rightarrow X$ be a mapping satisfying

$$
G(T x, T y, T y) \leq G(x, y, y)-\varphi(G(x, y, y))
$$

for all $x, y \in X$, where $\varphi:[0, \infty) \rightarrow[0, \infty)$ is continuous with $\varphi^{-1}(\{0\})=\{0\}$. Then $T$ has a unique fixed point.

Proof Consider the quasi-metric $\delta(x, y)=G(x, y, y)$ for all $x, y \in X$. From (3.12), we have

$$
d(T x, T y) \leq d(x, y)-\varphi(d(x, y))
$$

for all $x, y \in X$. Then the result follows from Theorem 3.2.

\section{Competing interests}

The authors declare that they have no competing interests.

\section{Authors' contributions}

All authors contributed equally and significantly to write this manuscript.

\section{Acknowledgements}

This work is supported by the Research Center, College of Science, King Saud University.

Received: 27 June 2012 Accepted: 2 November 2012 Published: 22 November 2012

\section{References}

1. Mustafa, Z: A new structure for generalized metric spaces-with applications to fixed point theory. PhD thesis, the University of Newcastle, Australia (2005)

2. Mustafa, Z, Sims, B: A new approach to generalized metric spaces. J. Nonlinear Convex Anal. 7(2), 289-297 (2006)

3. Mustafa, Z, Obiedat, H: A fixed point theorem of Reich in G-metric spaces. CUBO 12(1), 83-93 (2010)

4. Mustafa, Z, Shatanawi, W, Bataineh, M: Existence of fixed point results in G-metric spaces. Int. J. Math. Math. Sci. 2009, Article ID 283028 (2009)

5. Mustafa, Z, Sims, B: Fixed point theorems for contractive mappings in complete G-metric spaces. Fixed Point Theory Appl. 2009, Article ID 917175 (2009)

6. Shatanawi, W: Fixed point theory for contractive mappings satisfying $\Phi$-maps in G-metric spaces. Fixed Point Theory Appl. 2010, Article ID 181650 (2010)

7. Shatanawi, W: Some fixed point theorems in ordered G-metric spaces and applications. Abstr. Appl. Anal. 2011, Article ID 126205 (2011)

8. Mustafa, Z, Obiedat, H, Awawdeh, F: Some fixed point theorem for mapping on complete G-metric spaces. Fixed Point Theory Appl. 2008, Article ID 189870 (2008)

9. Ćirić, LB: A generalization of Banach's contraction principle. Proc. Am. Math. Soc. 45(2), 267-273 (1974)

10. Samet, B, Vetro, C, Vetro, F: Remarks on G-metric spaces. Int. J. Anal. (in press) 